\title{
Six-dimensional superconformal couplings of non-abelian tensor and hypermultiplets
}

\author{
Henning Samtleben, ${ }^{a}$ Ergin Sezgin $^{b}$ and Robert Wimmer ${ }^{a, c}$ \\ ${ }^{a}$ Université de Lyon, Laboratoire de Physique, UMR 5672, CNRS, \\ École Normale Supérieure de Lyon, 46, allée d'Italie, F-69364 Lyon cedex 07, France \\ ${ }^{b}$ George P. and Cynthia W. Mitchell Institute for Fundamental Physics and Astronomy, \\ Texas A $6 M$ University, College Station, TX 7r843-4242, U.S.A. \\ ${ }^{c}$ C.N. Yang Institute for Theoretical Physics, \\ State University of New York, Stony Brook, NY 11794-3840, U.S.A. \\ E-mail: henning.samtleben@ens-lyon.fr, sezgin@physics.tamu.edu, \\ wimmer@insti.physics.sunysb.edu
}

ABSTRACT: We construct six-dimensional superconformal models with non-abelian tensor and hypermultiplets. They describe the field content of $(2,0)$ theories, coupled to $(1,0)$ vector multiplets. The latter are part of the non-abelian gauge structure that also includes non-dynamical three- and four-forms. The hypermultiplets are described by gauged nonlinear sigma models with a hyper-Kähler cone target space. We also address the question of constraints in these models and show that their resolution requires the inclusion of abelian factors. These provide couplings that were previously considered for anomaly cancellations with abelian tensor multiplets and resulted in the selection of $A D E$ gauge groups.

Keywords: Brane Dynamics in Gauge Theories, Gauge Symmetry, Conformal Field Models in String Theory, M-Theory

ARXIV EPRINT: 1212.5199 


\section{Contents}

1 Introduction $\quad 1$

2 Geometrical setting $\quad 4$

$\begin{array}{llr}3 & \text { Superconformal Lagrangian } & 8\end{array}$

4 Eliminating auxiliary fields $\quad 14$

$\begin{array}{lll}5 & \text { Conclusions } & 17\end{array}$

$\begin{array}{lr}\text { A Conventions } & 18\end{array}$

$\begin{array}{ll}\text { B Target space geometry } & 19\end{array}$

\section{Introduction}

For a long time it was believed that four is the maximal dimension in which conformal field theories can exist. However, string/M-theory suggest that the group theoretical bound of six dimensions on superconformal theories [1] is actually saturated [2]. The understanding of effective $(2,0)$ theories for multiple M5-branes is one of the pressing questions of Mtheory in this context. They are classified by $A D E$ groups, but an explicit understanding still seems far off.

One of the problems is of pure geometrical origin and independent of any underlying dynamics or supersymmetry and addresses the question of non-abelian tensor (two-form) gauge fields. For example, various no-go theorems exclude the non-abelian extension of the abelian tensor gauge symmetry [3]. In [4] this problem was encompassed in the context of a tensor hierarchy $[5,6]$ by introducing additional form-degrees of freedom, in particular an ordinary gauge field and non-propagating three- and (optionally) four-form gauge fields. This structure shows similarity with concepts of higher gauge theories, Q structures, and non-abelian gerbes [7-12], extended to higher degree forms. A very particular realization of this gauge symmetry was given in [13].

The other problem is that the supposed $(2,0)$ theory of multiple M5-branes is intrinsically strongly coupled, i.e. it has no free parameter for a weak coupling expansion which would make the existence of a Lagrangian description plausible. This problem is analogous to the situation of M2-branes. Also in that case, and for the same reason, it was believed that a Lagrangian description does not exist. Nevertheless, a single maximally supersymmetric three-dimensional CFT (BLG-model) $[14,15]$ and a more general class with less supersymmetry (ABJM-models) [16] have been found. The decisive observation in the latter case is that by placing the M2-branes at an orbifold singularity instead of 
placing them in flat space one gains a dimensionless parameter which allows for a weak coupling limit and thus makes a Lagrangian description possible. The resulting CFT's have the same field content as a maximally, i.e. $\mathcal{N}=8$ supersymmetric theory but realize only $\mathcal{N}=6$ supersymmetry. From the field theory point of view this means that the reduced supersymmetry is less restrictive and therefore allows for a Lagrangian formulation.

In a similar spirit, in $[4,17]$ we constructed interacting $(1,0)$ superconformal models for non-abelian tensor and vector multiplets. The BPS sector of these models has been analyzed in [18]. Also in six dimensions, $\mathcal{N}=(1,0)$ supersymmetry is not as restrictive as to prevent any nontrivial local dynamics (as in the maximal $(2,0)$ case), but strong enough to essentially determine the dynamics. As a further step towards the sought-after $(2,0)$ models, in this paper we complete the $(1,0)$ tensor multiplet interactions to the full field content of the $(2,0)$ theories by coupling the non-abelian tensor/vector models of $[4,17]$ to superconformal hypermultiplets.

Superconformal hypermultiplets are described by a gauged non-linear sigma model [19]. Conformal invariance requires the target space to be a hyper-Kähler cone (HKC) [20, 21 . The possible gauge groups are subgroups of the isometry group of the underlying quaternionic Kähler manifold $(\mathrm{QK})$. Supersymmetry requires that the vector multiplets that gauge these isometries are embedded in a particular way into the vector multiplets of the superconformal vector/tensor system. There is no direct coupling between the tensor and hypermultiplets prior to eliminating the auxiliary fields, but the vector multiplets form the 'glue' between this two multiplets in a non-trivial way, beyond simple minimal gauge couplings. Though the restriction of the gauge group by the QK isometry group suggests some selection mechanism there remains a large freedom in the construction. Even the restriction to compact quaternionic Kähler manifolds provides for all classical groups a corresponding manifold, the Wolf spaces [22], and an associated HKC. We discuss in detail the case of the flat HKC and show that one can embed arbitrary matrix representations of semi-simple groups including abelian factors in the corresponding isometry group which in this case is $\operatorname{Sp}(n) / \mathbb{Z}_{2}$. It is however not surprising that pure classical considerations do not lead to the selection of $A D E$ groups since these are determined by anomaly cancellation conditions and is thus an essential quantum effect. For abelian tensor multiplets this was discussed in [23].

From the different types of superconformal tensor/vector models of [17] the coupling to the hypermultiplets selects those whose field equations can be integrated to an action (modulo the known subtleties related to the description of self-dual tensor fields). Upon extending the system to include non-propagating four-form potentials, the dynamics may equivalently be expressed by a set of non-abelian first-order duality equations. This description appears rather natural with the coupling to hypermultiplets. The resulting supermultiplet structure is rather intriguing. While the gauge structure based on the three-form leads to an on-shell supermultiplet that mixes the tensor and vector multiplet with the nondynamical three-form, the inclusion of the four-form also intertwines the hypermultiplets with the previous ones. Even more intriguing is the observation, that in the supersymmetry transformation of the four-form the tensor and hypermultiplet contributions combine in a manifestly $(2,0)$ way. 
Another question that we address in this paper is the elimination of the vector multiplet auxiliary fields. As has been mentioned, the vector multiplet forms the 'glue' between the tensor and hypermultiplet. A particular important coupling comes through the auxiliary field, which is described by the algebraic field equation,

$$
d_{\text {Irs }}\left(Y_{i j}^{s} \phi^{I}-2 \bar{\lambda}_{(i}^{s} \chi_{j)}^{I}\right)-\frac{1}{2 \lambda} \theta_{r}^{\mathfrak{m}} \mu_{\mathfrak{m}}^{i j}=0,
$$

where $Y$ is the auxiliary field and $\lambda_{i}^{s}$ the gaugino of the vector multiplet, while $\phi, \chi$ belong to the tensor multiplet. The moment maps $\mu$ of the hypermultiplet Lagrangian couple with a free dimensionless coupling constant $\lambda$. The other two objects are invariant tensors of the gauge group. This equation in fact contains the full information about the vector multiplet dynamics, the rest is fixed by supersymmetry. Generically this equation implies constraints on the elementary fields $[4,17]$. The inclusion of the hypermultiplets does not alter this observation. We show that one has to include abelian factors, or tensor multiplet singlets, in order to avoid constraints for the elementary fields. In that case one finds a unique solution for the auxiliary fields $Y$. In contrast to the standard YM-hyper couplings of [19] however, eliminating the auxiliary fields does not generate a bosonic potential but only quadratic and quartic fermionic interactions.

The resulting moduli space for the scalars is thus not constrained by any potential. In particular the VEV for the tensor scalar fields can in principle take any value. However, we find certain couplings between the tensor scalars and the vector multiplet for which the VEV $\langle\phi\rangle$ acts as the inverse (square) of the Yang-Mills coupling constant. Therefore at the conformal point $\langle\phi\rangle=0$ the theory is no longer perturbatively defined. Also the auxiliary field equation (1.1) is degenerate in this point. It implies constraints on the hypermultiplets and thus changes the target space geometry. This is of course the indication of the well known, but not so well understood, phenomenon of the tensionless string phase transition $[24,25]$. Consequently the models are well defined only on the Coulomb branch, where the conformal symmetry is spontaneously broken. However, since the breaking is spontaneous the original conformal symmetry might be still useful in the perturbative regime through the broken Ward identities, although they might look very complicated. The literature mentions evidence that this conformal fixed point might be described by a local field theory (see e.g. [23]). With the given set of degrees of freedom that we considered here, which comprises the full field content of $(2,0)$ tensor multiplets, superconformal symmetry predicts essentially unique models where this phenomenon can be at most seen as a highly non-perturbative effect.

A last comment regarding $A D E$ classification: $\mathcal{N}=(1,0)$ theories are chiral and therefore anomaly cancellation is an important ingredient. As mentioned above, the anomaly cancellation conditions of [23] lead to a selection of $A D E$ gauge groups, though the tensors are abelian in that case. For the models presented here, we have to postpone such a discussion until a full quantum treatment is available. However, we make the following observation: The necessity to include abelian factors to avoid constraints in (1.1) also provides the structure for the particular couplings that were considered in [23], see equation (4.18) below. These couplings, that are an essential input for the anomaly cancellation conditions, 
are thus naturally present in our theories. Let us finally mention other approaches towards the field equations $[11,26,27]$ and amplitudes [28] of the $(2,0)$ theory whose relation to the presented construction will be interesting to understand. We also want to mention possible relations two supergravity theories $[30,31]$

The paper is organized as follows: In section 2 we discuss the geometrical background for the superconformal hypermultiplets. In section 3 we describe the Lagrangians for the hyper- and the tensor/vector system, respectively, and the embedding of the hypermultiplet gauging into the latter. We also discuss the 'first-order' description, which includes the non-dynamical four-form and its supermultiplet structure. In section 4 we discuss the elimination of the auxiliary fields and the necessity of abelian factors and resulting interactions.

\section{Geometrical setting}

The target space of rigid supersymmetric sigma models with eight supercharges has to be a hyper-Kähler (HK) manifold ${ }^{1}\left(\mathcal{M}_{4 n}, g, \vec{J}\right)[33,34]$. Hence, $\mathcal{M}_{4 n}$ is a (real) $4 n$-dimensional, with local coordinates $q^{\alpha=1, \ldots, 4 n}$, and provides an $\operatorname{Sp}(1)$-triplet of covariant constant complex structures $\vec{J}$ and a metric $g$ which is hermitian w.r.t. all of them. This hyper-complex structure forms a quaternionic algebra and defines a triplet of hyper-Kähler forms $\vec{\omega}$ :

$$
J^{\mathrm{i}} J^{\mathrm{j}}=-\delta^{\mathrm{ij}}+\varepsilon^{\mathrm{ijk}} J^{\mathrm{k}}, \quad \nabla_{\alpha} \vec{J}=0, \quad \vec{\omega}_{\alpha \beta}:=g_{\alpha \gamma} \vec{J}_{\beta}^{\gamma},
$$

where $\nabla_{\alpha}$ is the Levi-Civita connection. This actually implies the existence of an whole $S^{2} \simeq \mathbb{C} P^{1}$ of complex structures $\left\{\mathcal{I}=\vec{a} \cdot \vec{J} \mid \vec{a}^{2}=1\right\}$, and that the Kähler forms are closed, $d \vec{\omega}=0$. The latter, or the requirement of reduced holonomy $\operatorname{Hol}(g) \subseteq \operatorname{Sp}(n)$ may be taken as an equivalent definition of a HK manifold. In the following we pick $J^{3}$ as the particular complex structure to define complex coordinates ${ }^{2} z^{a}, \bar{z}^{\bar{a}}=\left(z^{a}\right)^{*}$ such that $J^{3}$ is diagonal and the fundamental holomorphic $(2,0)$ form is given by:

$$
J_{b}^{3 a}=i \delta_{b}^{a}, \quad J^{3 \bar{a}_{\bar{b}}}=-i \delta^{\bar{a}_{\bar{b}}}, \quad \omega^{(+)}:=\frac{1}{2}\left(\omega^{1}+i \omega^{2}\right) .
$$

The holomorphic $(2,0)$ form $\omega^{(+)}$implies that a HK manifold is also a holomorphic symplectic manifold. Furthermore $\left(\omega^{(+)}\right)^{n}$ defines a nowhere vanishing section of the canonical bundle and thus $\mathcal{M}_{4 n}$ is Ricci-flat.

Hyper Kähler cones. For a sigma model with the given amount of supersymmetry to be conformal the HK target space $\mathcal{M}_{4 n}$ has to be of special type, namely a hyper-Kählercone (HKC) [20]. These spaces are characterized by the existence of a homothetic Killing vector field,

$$
\nabla_{\alpha} \chi^{\beta}=\delta_{\alpha}^{\beta} .
$$

This implies for the Lie derivative $\mathscr{L}_{\chi} g_{\alpha \beta}=2 g_{\alpha \beta}$ and thus $\chi^{\alpha}$ generates dilatations ${ }^{3}$ on $\mathcal{M}_{4 n}$, see also [35]. The homothetic Killing vector defines i.) a hyper-Kähler potential

\footnotetext{
${ }^{1}$ If one considers only equations of motions no metric $g$ is needed and the requirements for susy are less restrictive $[29,32]$. However, we will always assume the existence of an action.

${ }^{2}$ The real coordinates we define then as $\left[q^{\alpha}\right]=\frac{1}{\sqrt{2}}\left[\left(z^{a}+\bar{z}^{\bar{a}}\right),-i\left(z^{a}-\bar{z}^{\bar{a}}\right)\right]^{t}$.

${ }^{3}$ We define the homothetic Killing vector with unit normalization (2.3). The actual dilatation generator will be defined with a constant factor, appropriate for the six-dimensional world volume.
} 
$\chi(q): \mathcal{M}_{4 n} \rightarrow \mathbb{R}$, i.e. a single Kähler potential for all complex structures $\mathcal{I}$ [36], and ii.) the Killing vectors $\vec{k}^{\alpha}$ which generate the $\operatorname{Sp}(1)$ isometry that acts on the complex structures and becomes the R-symmetry of the supersymmetry algebra:

$$
\chi(q):=\frac{1}{2} g_{\alpha \beta} \chi^{\alpha} \chi^{\beta} \Leftrightarrow g_{\alpha \beta}=\nabla_{\alpha} \partial_{\beta} \chi(q), \quad \vec{k}^{\alpha}:=\frac{1}{2} \vec{J}^{\alpha}{ }_{\beta} \chi^{\beta},
$$

from which follows $\mathcal{L}_{\vec{\alpha} \vec{k}} J^{\mathrm{j}}=-\alpha^{\mathrm{i}} \varepsilon^{\mathrm{ijk}} J^{\mathrm{k}}=\vec{\alpha} \vec{t} \mathrm{j}_{\mathrm{k}} J^{\mathrm{k}}$. The equation for the metric can be considered as an equivalent definition of a HKC, where $\chi^{\alpha}$ is then obtained as $\chi^{\alpha}=g^{\alpha \beta} \partial_{\beta} \chi(q)$, and it imposes rather strong conditions. Not only are the non-vanishing components of the metric as usual derived from a Kähler potential (in complex coordinates $g_{a \bar{b}}=\partial_{a} \partial_{\bar{b}} \chi$ ), but it also implies that $\nabla_{a} \partial_{b} \chi=0$.

The superconformal case is closely related to the situation with local supersymmetry and the coupling to supergravity. The latter requires that the target space is a $4(n-1)$ dimensional quaternionic Kähler manifold $\mathcal{Q}_{4(n-1)}$ [37]. There exists a one-to-one correspondence between HKC's $\mathcal{M}_{4 n}$ and quaternionic Kähler manifolds $\mathcal{Q}_{4(n-1)}$ via the "superconformal quotient" [20,38] and the Swann bundle $\mathcal{M}_{4 n} \rightarrow \mathcal{Q}_{4(n-1)}$ [36]. The coupling to supergravity gauges the above described $\operatorname{Sp}(1)$ isometry. This is in contrast with the rigid superconformal case with global $\mathrm{Sp}(1) \mathrm{R}$-symmetry, that we consider here. However, we are interested in the construction of gauged superconformal models and the possible gauge groups are given by those isometries of the HKC $\mathcal{M}_{4 n}$ that can be gauged while preserving super- and conformal symmetry. These are the triholomorphic isometries which commute with the $\mathrm{Sp}(1)$ isometries (2.4) and the dilatations (2.3). As a matter of fact, these are the isometries of the underlying quaternionic Kähler manifold $\mathcal{Q}_{4(n-1)}[21,38]$. Thus Iso $\left(\mathcal{Q}_{4(n-1)}\right)$ describes the possible gauge groups of the superconformal sigma models with the corresponding HKC target space $\mathcal{M}_{4 n} \rightarrow \mathcal{Q}_{4(n-1)}$.

It is conjectured [39] that the Wolf spaces [22] are all possible (positive curvature) compact quaternionic manifolds $\mathcal{Q}_{4(n-1)}$. These are symmetric spaces and there exists one for each simple Lie group. The quaternionic projective space,

$$
\mathcal{Q}_{4(n-1)}=\mathbb{H} P^{n-1}=\frac{\operatorname{Sp}(n)}{\operatorname{Sp}(1) \times \operatorname{Sp}(n-1)},
$$

whose HKC is the flat space $\mathbb{R}^{4 n}$, will be of particular interest for us. The isometry group in this case is $\operatorname{Sp}(n) / \mathbb{Z}_{2}[40]$ and thus we can realize representations of gauge groups that can be embedded in in $\operatorname{Sp}(n) / \mathbb{Z}_{2}$. For the rest of this paper, we will restrict to discussing gaugings on the level of the Lie algebra, i.e. for $\operatorname{Sp}(n)$.

Triholomorphic isometries, moment maps. As has been indicated, the isometries that can be gauged in accordance with superconformal symmetry have to be i.) triholomorphic and commute with the $\mathrm{Sp}(1)$ isometries in order to preserve supersymmetry [34, 41], and ii.) commute with the homothetic Killing vector field in order to preserve conformal invariance [38]. Therefore the corresponding Killing vector fields $X_{(\hat{\mathfrak{m}})}$ are defined by the conditions,

$$
\mathscr{L}_{X_{(\mathfrak{m})}} g_{\alpha \beta}=0, \quad \mathscr{L}_{X_{(\hat{\mathfrak{m}})}} \vec{\omega}_{\alpha \beta}=0, \quad\left[X_{(\hat{\mathfrak{m}})}, \vec{k}\right]=\left[X_{(\hat{\mathfrak{m}})}, k_{D}\right]=0,
$$


where we have introduced the properly normalized dilatation Killing vector $k_{D}^{\alpha}=w_{q} \chi^{A}$, with $w_{q}=2$ for a six-dimensional world volume. Given that the Kähler forms $\vec{\omega}$ are closed the triholomorphicity condition reduces to $\mathscr{L}_{X_{(\hat{\mathfrak{m}})}} \vec{\omega}=d\left(i_{X_{(\hat{\mathfrak{m}})}} \vec{\omega}\right)=0$. This in turn implies the existence of $\mathrm{Sp}(1)$-triplets of (local) moment maps (Killing potentials) $\vec{\mu}_{(\hat{\mathfrak{m}})}$,

$$
d \vec{\mu}_{(\hat{\mathfrak{m}})}:=-i_{X_{(\mathfrak{\mathfrak { m }})} \vec{\omega}} \overrightarrow{\partial_{\alpha}} \vec{\mu}_{(\hat{\mathfrak{m}})}=\vec{\omega}_{\alpha \beta} X_{(\mathfrak{\mathfrak { m }})}^{\beta}
$$

which reflects the mentioned symplectic structure of the HK manifold. The moment maps $\vec{\mu}_{(\hat{\mathfrak{m}})}$ will define the potential-coupling to the vector multiplet, that one obtains after gauging the isometries. Therefore the global existence of the moment maps is a necessary (and sufficient [42]) condition to gauge the associated isometries. The moment maps (2.7) are defined only up to constants, which however are eventually fixed by the requirement of conformal symmetry, see (B.14).

Gauging. The gauging of isometries, especially in the given context, has been considered in [19-21, 41]. The triholomorphic Killing vector fields $X_{(\hat{\mathfrak{m}})}$ generate the isometry group $\hat{G}=\operatorname{Iso}\left(\mathcal{Q}_{4(n-1)}\right)$ of the quaternionic Kähler manifold associated to the HKC $\mathcal{M}_{4 n}$. Generically we will gauge a subgroup $G \subseteq \hat{G}$, generated by the subset of triholomorphic isometries $\left\{X_{(\mathfrak{m})}\right\} \subseteq\left\{X_{(\hat{\mathfrak{m}})}\right\}$ that satisfy,

$$
\left[X_{(\mathfrak{m})}, X_{(\mathfrak{n})}\right]=-f_{\mathfrak{m} \mathfrak{n}}^{\mathfrak{p}} X_{(\mathfrak{p})}
$$

The $f_{\mathfrak{m n}}{ }^{\mathfrak{p}}$ are the structure constants of the associated Lie algebra $\mathfrak{g}:=\operatorname{Lie}(G)$.

The target space coordinates $q^{\alpha}$ will eventually depend on the six-dimensional world volume coordinates $x^{\mu}$. In the process of gauging the isometry transformations are made local w.r.t. the world volume:

$$
\delta_{\Lambda} q^{\alpha}(x):=\Lambda^{\mathfrak{m}}(x) X_{(\mathfrak{m})}^{\alpha} .
$$

Correspondingly one introduces gauge fields on the world-volume and covariant derivatives,

$$
D_{\mu} q^{\alpha}=\partial_{\mu} q^{\alpha}-A_{\mu}^{\mathfrak{m}} X_{(\mathfrak{m})}^{\alpha}, \quad \delta_{\Lambda} A_{\mu}^{\mathfrak{m}}=\partial_{\mu} \Lambda^{\mathfrak{m}}-A_{\mu}^{\mathfrak{n}} \Lambda^{\mathfrak{p}} f_{\mathfrak{n p}}^{\mathfrak{m}}
$$

which results in the covariant transformation law,

$$
\delta_{\Lambda}\left(D_{\mu} q^{\alpha}\right)=\Lambda^{\mathfrak{m}}\left(T_{\mathfrak{m}}\right)^{\alpha}{ }_{\beta} D_{\mu} q^{\beta} \quad \text { with } \quad\left(T_{\mathfrak{m}}\right)^{\alpha}{ }_{\beta}=\partial_{\beta} X_{(\mathfrak{m})}^{\alpha}
$$

We finally note that in the case that $G \subset \hat{G}$ is a normal subgroup the original global isometry group is preserved by the gauging and one has in addition to the gauge invariance $G$ the global symmetry $H=\hat{G} / G$. However, in the generic case the original symmetry $\hat{G}$ is broken down to $G$ by the gauging [19].

Flat target space, $\operatorname{Sp}(\boldsymbol{n})$ isometries. We conclude this section with an explicit discussion for the case that the target space is flat $\mathbb{R}^{4 n}$ which will be the case of particular interest in the following. Nevertheless, all of the subsequent constructions apply to general HKC target spaces. For flat target space, all connection coefficients can be set to zero 
and also the curvature tensor in (3.3) vanishes. The basic HK structures take in complex coordinates the simple form,

$$
\chi=\sum_{a=1}^{2 n}\left|z^{a}\right|^{2}, \quad \omega^{3}=-i d z^{a} \wedge d z^{\bar{a}}, \quad \omega^{(+)}=\frac{1}{2} \Omega_{a b} d z^{a} \wedge d z^{b},
$$

with the $\operatorname{Sp}(n)$ invariant symplectic form $\Omega_{a b}(a, b=1, \ldots, 2 n)$, while the homothetic/dilatation and $\mathrm{Sp}(1)$ Killing vectors are given by,

$$
\chi^{a}=\frac{1}{2} k_{D}^{a}=z^{a}, \quad k^{3 a}=\frac{1}{2} z^{a}, k^{+a}=0, k^{-a}=\frac{1}{2}(\Omega \bar{z})^{a} .
$$

The conjugate components of the vectors are simply obtained by complex conjugation, $k^{3 \bar{a}}=\left(k^{3 a}\right)^{*}, k^{+\bar{a}}=\left(k^{-a}\right)^{*}$, etc.. For the resulting triholomorphic isometries (2.6) and moment maps (2.7) one obtains,

$$
\begin{aligned}
& X_{(\hat{\mathfrak{m}})}^{a}=u_{(\hat{\mathfrak{m}})}^{a} b^{b} z^{b} \quad \text { with } \quad u_{(\hat{\mathfrak{m}})}^{\dagger}=-u_{(\hat{\mathfrak{m}})} \text { and } u_{(\hat{\mathfrak{m}})}^{t}=\Omega u_{(\hat{\mathfrak{m}})} \Omega, \\
& \mu_{(\hat{\mathfrak{m}})}^{3}=i\left(\bar{z} u_{(\hat{\mathfrak{m}})} z\right), \quad \mu_{(\hat{\mathfrak{m}})}^{(+)}=\frac{1}{2}\left(\Omega u_{(\hat{\mathfrak{m}})}\right)_{a b} z^{a} z^{b} .
\end{aligned}
$$

The matrices $u_{(\hat{\mathfrak{m}})}$ thus generate the group $\operatorname{Sp}(n)$, which is the isometry group of the underlying quaternionic manifold $\mathbb{H} P(2.5)$. It is easy to see that all isometries commute: $\left[X_{\hat{\mathfrak{m}}}, k_{D}\right]=\left[X_{\hat{\mathfrak{m}}}, \vec{k}\right]=\left[\vec{k}, k_{D}\right]=0$. This would be not the case for the translational triholomorphic isometries of $\mathbb{R}^{4 n}$. However, the $\operatorname{Sp}(1) \mathrm{R}$-isometry is obviously not manifestly realized on the complex coordinates $z^{a}$, since it rotates the complex structures (2.4).

In order to realize the $\mathrm{Sp}(1) \mathrm{R}$-isometry in a manifest way we introduce the pseudoreal coordinates,

$$
q^{i a}=\left[\begin{array}{l}
q^{1} \\
q^{2}
\end{array}\right]:=\kappa\left[\begin{array}{c}
\Omega \bar{z} \\
-i z
\end{array}\right]=f_{\alpha}^{i a} q^{\alpha} \Rightarrow\left(q^{i a}\right)^{*}=\varepsilon_{i j} \Omega_{a b} q^{j b},
$$

where $\kappa$ is a phase such that $\kappa^{2}=i$. The constant flat vielbeine, $f^{i a}{ }_{\alpha}, f^{\alpha}{ }_{i a}$ are given explicitly in (B.1). Dilatations, $\operatorname{Sp}(1)$ and $\operatorname{Sp}(n)$ action on these coordinates are of the covariant form,

$$
\delta_{\lambda k_{D}} q^{i a}=2 \lambda q^{i a}, \quad \delta_{\vec{\alpha} \vec{k}} q^{i a}=\vec{\alpha} \vec{t}^{i}{ }_{j} q^{i b}, \quad \delta_{\lambda^{\hat{\mathfrak{m}}} X_{(\hat{\mathfrak{m}})}} q^{i a}=\lambda^{\hat{\mathfrak{m}}} u_{(\hat{\mathfrak{m}})}{ }^{a}{ }_{b} q^{i b},
$$

where $\vec{t}^{i}{ }_{j}=-\frac{i}{2} \vec{\sigma}^{i}{ }_{j}$. The coordinates $q^{i a}$ thus transform in the fundamental representation $(\mathbf{2}, \mathbf{2 n})$ under $\operatorname{Sp}(1) \times \operatorname{Sp}(n)$. The basic data for the HKC and the Lagrangian (3.3) are given by

$$
\begin{aligned}
g & =\varepsilon_{i j} \Omega_{a b} d q^{i a} \otimes d q^{j b}, & \omega^{i j} & =\Omega_{a b} d q^{i a} \wedge d q^{j b}, \\
X_{(\mathfrak{\mathfrak { m }})}^{i a} & =u_{(\hat{\mathfrak{m}}){ }_{b}{ }^{a} q^{i b},} & \mu_{(\hat{\mathfrak{m}})}^{i j} & =\left(\Omega u_{(\hat{\mathfrak{m}})}\right)_{a b} q^{i a} q^{j b} .
\end{aligned}
$$

The next step is to choose subgroups $G \subseteq \operatorname{Sp}(n)$ and their representations which can be embedded in the $s p(n)$ matrices $u_{(\hat{\mathfrak{m}})}$. Denoting the corresponding matrices by $u_{(\mathfrak{m})}$ one finds from (2.8), (2.15),

$$
\left[u_{(\mathfrak{m})}, u_{(\mathfrak{n})}\right]=f_{\mathfrak{m n}}^{\mathfrak{p}} u_{(\mathfrak{p})}
$$


ADE embeddings. We choose a canonical form for the $2 n \times 2 n$ component matrix $\Omega$ of the symplectic form (3.1), (2.12), which then specifies the general structure of any $s p(n)$ matrix $u(2.14)$ :

$$
\Omega=\left[\begin{array}{r}
1 \\
-\mathbb{1}
\end{array}\right] \Rightarrow u=\left[\begin{array}{cc}
A & B \\
-B^{*} & -A^{t}
\end{array}\right] \text { with: } A^{\dagger}=-A, B^{t}=B .
$$

This allows for diverse embeddings of different groups. For example with $B=0$ one obtains the embedding $\mathrm{U}(n) \hookrightarrow \mathrm{Sp}(n)$ with two copies of the hypermultiplets with the second in the contragredient representation of the first one. A different $\mathrm{U}(n)=\mathrm{SO}(2 n) \cap \mathrm{Sp}(n) \hookrightarrow \operatorname{Sp}(n)$ embedding is obtained by by choosing $A$ and $B$ to be real. If one sets $B=0$ in the latter case one obtains the embedding $\mathrm{SO}(n) \hookrightarrow \mathrm{Sp}(n)$, again with two copies of hypermultiplets, one in the contragredient representation of the other. It is thus clear that one can embed all classical groups, including abelian factors, and taking the dimension $n$ large enough any representation therof.

This means that at this stage there is no restriction to $A D E$ gauge groups as one would expect for effective theories of multiple $M 5$-branes. However, it is not to be expected to happen at the classical level. In terms of effective CFT's the restriction to $A D E$ gauge groups results from anomaly cancelation conditions [23], and is thus an essential quantum effect. We have to postpone such a discussion in the context of our models to subsequent investigation.

\section{Superconformal Lagrangian}

Hypermultiplets. Supersymmetry requires the tangent bundle of the hyper-Kähler cone $\mathcal{M}_{4 n}$ to be of the form $T \mathcal{M}_{(4 n)}=\mathrm{H}_{\mathrm{Sp}(1)} \otimes \mathrm{P}_{\mathrm{Sp}(n)}$ [37], hence the structure group is $\mathrm{Sp}(1) \times \mathrm{Sp}(n)$. The sections of the trivial (pull-back) bundle $\mathrm{H}_{\mathrm{Sp}(1)}$ are the constant susy parameters $\epsilon^{i}$ and thus the $\operatorname{Sp}(n)$ bundle $\mathrm{P}_{\mathrm{Sp}(n)}$ defines the holonomy group. This gives the mentioned HK condition $\operatorname{Hol}(g) \subseteq \operatorname{Sp}(n)$. Consequently there exists a local vielbein $f^{\alpha}{ }_{i a}$ and its inverse $f^{i a}{ }_{\alpha}$ which satisfy the vielbein postulate and provide an expression for the metric:

$$
\begin{aligned}
& g_{\alpha \beta}=\varepsilon_{i j} \Omega_{a b} f_{\alpha}^{i a} f^{j b}{ }_{\beta} \quad \Leftrightarrow \quad g_{\alpha \beta} f^{\alpha}{ }_{i a} f^{\beta}{ }_{j b}=\varepsilon_{i j} \Omega_{a b}, \\
& \nabla_{\alpha} f^{i a}{ }_{\beta}+\omega_{\alpha}{ }_{b} f^{i b}{ }_{\beta}=0 \quad \Leftrightarrow \quad \delta^{i}{ }_{j} \omega_{\alpha b}{ }^{a}=f^{i a}{ }_{\beta} \nabla_{\alpha} f^{\beta}{ }_{j b} .
\end{aligned}
$$

Here, $\omega_{\alpha}{ }^{a} b$ is the $\operatorname{Sp}(n)$-connection. We collect further properties of HK manifolds in appendix B.

The susy transformations for the hypermultiplets are given by [19, 29],

$$
\begin{aligned}
\delta q^{\alpha} & =f^{\alpha}{ }_{i a} \bar{\epsilon}^{i} \psi^{a}, \\
\delta \psi^{a} & =\frac{1}{2} \not D q^{\alpha} \epsilon_{i} f_{\alpha}^{i a}-\delta q^{\alpha} \omega_{\alpha}{ }^{a}{ }_{b} \psi^{b},
\end{aligned}
$$

where $D_{\mu}$ is the covariant derivative (2.10). The dilatation and special supersymmetry transformations will be given below. The conformally supersymmetric Lagrangian for 
the gauged sigma model coupled to the off-shell vector multiplet $\left(A_{\mu}^{\mathfrak{m}}, \lambda^{i \mathfrak{m}}, Y_{i j}^{\mathfrak{m}}\right)$ can be obtained by restricting the general supersymmetric model of [19] to a HKC target space and the gauging of isometries satisfying (2.6). The resulting Lagrangian is ${ }^{4}$

$$
\begin{aligned}
\mathcal{L}_{\text {hyp }}=- & \frac{1}{2} g_{\alpha \beta} D_{\mu} q^{\alpha} D^{\mu} q^{\beta}+\bar{\psi}_{a} \gamma^{\mu} \mathscr{D}_{\mu} \psi^{a}-\frac{1}{8} W_{a b c d} \bar{\psi}^{a} \gamma^{\mu} \psi^{b} \bar{\psi}^{c} \gamma_{\mu} \psi^{d} \\
& +4 \bar{\psi}_{a} \bar{\lambda}_{i}^{\mathfrak{m}} f^{i a}{ }_{\alpha} X_{(\mathfrak{m})}^{\alpha}+Y_{i j}^{\mathfrak{m}} \mu_{(\mathfrak{m})}^{i j},
\end{aligned}
$$

where $X_{(\mathfrak{m})}^{\alpha}$ are the triholomorphic Killing vectors (2.8) and $\mu_{(\mathfrak{m})}{ }^{i}{ }_{j}=i \vec{\sigma}^{i}{ }_{j} \vec{\mu}_{(\mathfrak{m})}$ are the associated moment maps (2.7). The $\operatorname{Sp}(n)$ curvature tensor $W_{a b c d}$ is defined in (B.7). The gauge covariant derivative for the fermions is given by

$$
\mathscr{D}_{\mu} \psi^{a} \equiv \partial_{\mu} \psi^{a}-A_{\mu}^{\mathfrak{m}} t_{(\mathfrak{m})}{ }^{a} b \psi^{b}+\partial_{\mu} q^{\alpha} \omega_{\alpha}^{a} b \psi^{b},
$$

where $t_{(\mathfrak{m})}{ }^{a} b$ is defined as $t_{(\mathfrak{m})}{ }^{a}{ }_{b}=\frac{1}{2} f^{i a}{ }_{\alpha} \nabla_{\beta} X_{(\mathfrak{m})}{ }^{\alpha} f^{\beta}{ }_{i b}$, and satisfies the algebra (2.18). Under gauge transformations, (3.4) transforms as

$$
\delta_{\Lambda} \psi^{a}=L_{b}^{a} \psi^{b}, \quad \delta_{\Lambda}\left(\mathscr{D}_{\mu} \psi^{a}\right)=L^{a}{ }_{b} \mathscr{D}_{\mu} \psi^{b}, \quad L^{a}{ }_{b} \equiv\left[\Lambda^{\mathfrak{m}} t_{(\mathfrak{m})}{ }^{a}{ }_{b}-\delta_{\Lambda} q^{\alpha} \omega_{\alpha}{ }^{a} b\right] .
$$

Finally, we recall the standard off-shell supersymmetry transformations of the vector multiplet:

$$
\delta A_{\mu}^{\mathfrak{m}}=-\bar{\epsilon} \gamma_{\mu} \lambda^{\mathfrak{m}}, \quad \delta \lambda^{i \mathfrak{m}}=\frac{1}{8} \gamma^{\mu \nu} F_{\mu \nu}^{\mathfrak{m}} \epsilon^{i}-\frac{1}{2} Y^{i j \mathfrak{m}} \epsilon_{j}, \quad \delta Y^{i j \mathfrak{m}}=-\bar{\epsilon}^{(i} \not D \lambda^{j) \mathfrak{m}}
$$

Tensor-vector multiplet. The Lagrangian (3.3) is supersymmetric under (3.2) and the standard off-shell susy transformation rules (3.6) for the vector multiplet. The gauging should be accompanied by kinetic terms for the vector multiplet, however the pure YangMills action is not conformally invariant in six dimensions. To achieve conformal invariance a compensating supermultiplet is needed. This role can be played by a collection of tensor multiplets and the corresponding model has been constructed in [4]. The field content of this conformal tensor-vector model is given by a set of $n_{\mathrm{T}}$ tensor multiplets $\left\{\phi^{I}, B_{\mu \nu}^{I}, \chi^{I}\right\}$ and $n_{\mathrm{V}}$ vector multiplets $\left\{A_{\mu}^{r}, \lambda^{r}, Y_{i j}^{r}\right\}$, labeled by indices $I$ and $r$, respectively. In addition, the model in its most general form requires the introduction of the non-propagating 3form potentials $C_{\mu \nu \rho r}$. The vectors $A^{\mathfrak{m}}$ gauging the HKC isometries, cf. (2.10), and its superpartners will be identified with a subset of these fields below. To begin with, we shall recall the superconformal invariant interactions of these multiplets that admit an action formulation (modulo the standard subtleties of actions for self-dual tensor fields), as constructed in [4] to which we refer for details. We shall then discuss the superconformal invariance of the total action which also includes the hypermultiplets.

For vector and two-form tensor fields, the full covariant non-abelian field strengths are given by

$$
\begin{aligned}
\mathcal{F}_{\mu \nu}^{r} & \equiv 2 \partial_{[\mu} A_{\nu]}^{r}-f_{s t}{ }^{r} A_{\mu}^{s} A_{\nu}^{t}+h^{r}{ }_{I} B_{\mu \nu}^{I} \\
\mathcal{H}_{\mu \nu \rho}^{I} & \equiv 3 D_{[\mu} B_{\nu \rho]}^{I}+6 d^{I}{ }_{r s} A_{[\mu}^{r} \partial_{\nu} A_{\rho]}^{s}-2 f_{p q}{ }^{s} d_{r s}^{I} A_{[\mu}^{r} A_{\nu}^{p} A_{\rho]}^{q}+h^{r I} C_{\mu \nu \rho r},
\end{aligned}
$$

\footnotetext{
${ }^{4}$ By a rescaling of the vector multiplet $\left(A^{\mathfrak{m}}, \lambda^{\mathfrak{m} i}, Y_{i j}^{\mathfrak{m}}\right)$ (and associated gauge parameters $\Lambda^{\mathfrak{m}}$ ) one may introduce an explicit coupling constant.
} 
in terms of the antisymmetric structure constants $f_{s t}{ }^{r}=f_{[s t]}{ }^{r}$, a symmetric $d$-symbol $d_{r s}^{I}=d_{(r s)}^{I}$, and the tensor $h^{r}{ }_{I}$ inducing general Stückelberg-type couplings among forms of different degree. Indices $I, J$ are raised and lowered with a constant symmetric tensor $\eta_{I J}$. The covariant derivatives are defined as $D_{\mu} \equiv \partial_{\mu}-A_{\mu}^{r} X_{r}$. The field strengths (3.7) are defined such that they transform covariantly under the set of nonabelian gauge transformations

$$
\begin{aligned}
\delta A_{\mu}^{r} & =D_{\mu} \Lambda^{r}-h^{r}{ }_{I} \Lambda_{\mu}^{I} \\
\Delta B_{\mu \nu}^{I} & =2 D_{[\mu} \Lambda_{\nu]}^{I}-2 d_{r s}^{I} \Lambda^{r} \mathcal{F}_{\mu \nu}^{s}-h^{r I} \Lambda_{\mu \nu r}, \\
h^{r}{ }_{I} \Delta C_{\mu \nu \rho r} & =h^{r}{ }_{I}\left(3 D_{[\mu} \Lambda_{\nu \rho] r}+6 d_{I r s} \mathcal{F}_{[\mu \nu}^{s} \Lambda_{\rho]}^{I}+2 d_{I r s} \mathcal{H}_{\mu \nu \rho}^{I} \Lambda^{s}\right),
\end{aligned}
$$

where we have used the compact notation

$$
\begin{aligned}
\Delta B_{\mu \nu}^{I} & \equiv \delta B_{\mu \nu}^{I}-2 d_{r s}^{I} A_{[\mu}^{r} \delta A_{\nu]}^{s}, \\
\Delta C_{\mu \nu \rho r} & \equiv \delta C_{\mu \nu \rho r}-6 d_{I r s} B_{[\mu \nu}^{I} \delta A_{\rho]}^{s}-4 d_{I r s} d_{p q}^{I} A_{[\mu}^{s} A_{\nu}^{p} \delta A_{\rho]}^{q} .
\end{aligned}
$$

This tensor/vector gauge system is completely defined by the choice of the constant tensors $h^{r}{ }_{I}, d_{r s}^{I}, f_{r s}{ }^{t}$. Consistency of the tensor hierarchy, i.e. covariance of the field strengths (3.7) requires that the gauge group generators in the various representations are given by

$$
\begin{aligned}
\left(X_{r}\right)_{s}^{t} & =\left(X_{r}^{\mathrm{V}}\right)_{s}^{t} \equiv-f_{r s}{ }^{t}+h^{t}{ }_{I} d_{r s}^{I}, \\
\left(X_{r}\right)_{I}{ }^{J} & =\left(X_{r}^{\mathrm{T}}\right)_{I}{ }^{J} \equiv 2 d^{J}{ }_{r s} h^{s}{ }_{I}-2 h^{s J} d_{I s r},
\end{aligned}
$$

in terms of the constant tensors parametrizing the system. Further constraints on these tensors follow from closure of the algebra (or generalized Jacobi identities)

$$
\left[X_{r}, X_{s}\right]=-\left(X_{r}\right)_{s}^{t} X_{t}
$$

and gauge invariance of the tensor $d^{I}{ }_{r s}$, see [4] for the explicit form of these constraints. The 'action' describing the superconformal invariant couplings of the non-abelian tensor/vector system is given by

$$
\begin{aligned}
\mathcal{L}_{\mathrm{VT}}= & \frac{1}{8} d_{\text {Irs }} \phi^{I}\left(\mathcal{F}_{\mu \nu}^{r} \mathcal{F}^{\mu \nu s}-4 Y_{i j}^{r} Y^{i j s}+8 \bar{\lambda}^{r} \not D \lambda^{s}\right)-\frac{1}{8} D^{\mu} \phi_{I} D_{\mu} \phi^{I}-\frac{1}{2} \bar{\chi}_{I} \not D \chi^{I} \\
& -\frac{1}{96} \mathcal{H}_{\mu \nu \rho}^{I} \mathcal{H}_{I}^{\mu \nu \rho}-\frac{1}{24} d_{I r s} \mathcal{H}_{\mu \nu \rho}^{I} \bar{\lambda}^{r} \gamma^{\mu \nu \rho} \lambda^{s}-\frac{1}{2} d_{I r s}\left(\mathcal{F}_{\mu \nu}^{r} \bar{\lambda}^{s} \gamma^{\mu \nu} \chi^{I}-4 Y_{i j}^{r} \bar{\lambda}^{i s} \chi^{j I}\right) \\
& +\left(d_{J s r} h_{I}^{s}-4 d_{I s r} h_{J}^{s}\right) \phi^{I} \bar{\lambda}^{r} \chi^{J}+\frac{1}{4} d_{\text {Irs }} h^{r}{ }_{J} h_{K}^{s} \phi^{I} \phi^{J} \phi^{K} \\
& -\frac{1}{6} d_{I r s} d_{u v}^{I} \bar{\lambda}^{r} \gamma^{\mu} \lambda^{u} \bar{\lambda}^{s} \gamma_{\mu} \lambda^{v}-\frac{1}{48} \mathcal{L}_{\text {top }}
\end{aligned}
$$

where the topological term $\mathcal{L}_{\text {top }}$ is given by integrating

$$
d V \delta \mathcal{L}_{\text {top }}=6\left\{2 d_{\text {Irs }} \delta A^{r} \wedge \mathcal{F}^{s} \wedge \mathcal{H}^{I}-\Delta B^{I} \wedge\left(h^{r}{ }_{I} \mathcal{H}_{r}^{(4)}-d_{I r s} \mathcal{F}^{r} \wedge \mathcal{F}^{s}\right)-h^{r}{ }_{I} \Delta C_{r} \wedge \mathcal{H}^{I}\right\},
$$


with $\mathcal{H}^{(4)}$ defined in (3.23) below. Finally, it is important to note that for the tensor multiplet, this action has to be supplemented with the first-order self-duality equation

$$
\mathcal{H}_{\mu \nu \rho}^{I-}=-d_{r s}^{I} \bar{\lambda}^{r} \gamma_{\mu \nu \rho} \lambda^{s}
$$

to be imposed after having derived the second-order equations of motion. This is due to the well known fact that $p$-form potentials with self-dual field strengths do not admit a manifestly Lorentz covariant action formulation. Moreover, it has been observed in [4] that the metric $\eta_{I J}$ defining the kinetic terms in the tensor sector is necessarily of indefinite signature, i.e. a priori the spectrum of the Lagrangian (3.12) contains ghosts whose fate will require a more extensive analysis.

The action of the above Lagrangian is invariant under the following supersymmetry transformations

$$
\begin{aligned}
\delta A_{\mu}^{r} & =-\bar{\epsilon} \gamma_{\mu} \lambda^{r} \\
\delta \lambda^{i r} & =\frac{1}{8} \gamma^{\mu \nu} \mathcal{F}_{\mu \nu}^{r} \epsilon^{i}-\frac{1}{2} Y^{i j r} \epsilon_{j}+\frac{1}{4} h^{r}{ }_{I} \phi^{I} \epsilon^{i} \\
\delta Y^{i j r} & =-\bar{\epsilon}^{(i} \not D \lambda^{j) r}+2 h^{r}{ }_{I} \bar{\epsilon}^{(i} \chi^{j) I} \\
\delta \phi^{I} & =\bar{\epsilon} \chi^{I} \\
\delta \chi^{i I} & =\frac{1}{48} \gamma^{\mu \nu \rho} \mathcal{H}_{\mu \nu \rho}^{I} \epsilon^{i}+\frac{1}{4} \not D \phi^{I} \epsilon^{i}+\frac{1}{2} d^{I}{ }_{r s} \bar{\lambda}^{i r} \gamma^{\mu} \lambda^{j s} \gamma_{\mu} \epsilon_{j} \\
\Delta B_{\mu \nu}^{I} & =-\bar{\epsilon} \gamma_{\mu \nu} \chi^{I}, \\
h^{r}{ }_{I} \Delta C_{\mu \nu \rho r} & =-2 h^{r}{ }_{I} d_{J r s}\left(\bar{\epsilon} \gamma_{\mu \nu \rho} \lambda^{s} \phi^{J}\right) .
\end{aligned}
$$

Next, we turn to the description of the full action describing the superconformal couplings of the hypermultiplets to the non-abelian tensor/vector system.

The full Lagrangian. To put together the actions for the hypermultiplet and the tensor/vector system, we need to identify the vectors $A_{\mu}^{\mathfrak{m}}$ used to gauge the HKC isometries, cf. (2.10), as well as its superpartners, as a subset of the the vectors $A_{\mu}^{r}$ and their superpartners according to

$$
A^{\mathfrak{m}}=A^{r} \theta_{r}^{\mathfrak{m}}, \quad \lambda^{\mathfrak{m}}=\lambda^{r} \theta_{r}^{\mathfrak{m}}, \quad Y_{i j}^{\mathfrak{m}}=Y_{i j}^{r} \theta_{r}{ }^{\mathfrak{m}},
$$

with the constant embedding tensor $\theta_{r}{ }^{\mathfrak{m}}$. For consistency, the embedding of the vector fields (3.15) has to be supplemented with the following constraints on the embedding tensor:

$$
h^{r}{ }_{I} \theta_{r}^{\mathfrak{m}}=0, \quad f_{r s}{ }^{t} \theta_{t}^{\mathfrak{m}}=\theta_{r}^{\mathfrak{n}} \theta_{s}^{\mathfrak{p}} f_{\mathfrak{n p}}^{\mathfrak{m}} .
$$

The first condition guarantees that the modification of the susy transformations (3.14) does not affect the variation of the hypermultiplet action. Whereas the second condition guarantees that the embedding is homomorphic. The full Lagrangian is given by the sum

$$
\mathcal{L}=\mathcal{L}_{\text {VT }}+\frac{1}{2 \lambda} \mathcal{L}_{\text {hyp }} .
$$


where we introduced a relative (dimensionless) coupling constant $\lambda$. Both Lagrangians are separately supersymmetric so that $\lambda$ is a free parameter.

The fact that both actions are separately supersymmetric requires the first of the conditions in (3.16). Note also that while the field equations of the tensor multiplets do not involve the hypermultiplet, those of the vector- and hypermultiplets evidently mix. The invariance of the total action guaranties the fact that all the field equation transform into each other under supersymmetry. Nonetheless, given the fact that the field equation for the auxiliary field $Y_{r}^{i j}$ contains a contribution coming from the moment map of the hypermultiplet sector, it is instructive to examine how the vector multiplet field equations behave under supersymmetry. These field equations take the form

$$
\begin{array}{ll}
\delta Y_{r}^{i j}: & \mathcal{E}_{r}^{i j}+\frac{1}{2 \lambda} \theta_{r}^{\mathfrak{m}} \mu_{(\mathfrak{m})}^{i j}=0, \\
\delta \lambda^{i r}: & \mathcal{E}_{r}^{i}+\frac{2}{\lambda} \theta_{r}^{\mathfrak{m}} X_{(\mathfrak{m})}^{\alpha} f_{\alpha}^{i a} \psi_{a}=0, \\
\delta A_{\mu}^{r}: & \mathcal{E}_{\mu r}+\frac{1}{2 \lambda} \theta_{r}^{\mathfrak{m}}\left(D_{\mu} q^{\alpha} X_{(\mathfrak{m}) \alpha}-t_{(\mathfrak{m})}{ }_{b} b \bar{\psi}_{a} \gamma_{\mu} \psi^{b}\right)=0,
\end{array}
$$

where $\mathcal{E}_{r}^{i j}, \mathcal{E}_{r}^{i}$ and $\mathcal{E}_{\mu r}$ represent the contributions from the Lagrangian $\mathcal{L}_{\mathrm{VT}}$ to the field equations of the vector multiplet. These contributions are established to transform into each other in [4], and one can check that the supersymmetry variation of the contributions multiplying the embedding tensor $\theta_{r}{ }^{\mathfrak{m}}$ also transform into each other, as expected.

Higher $\boldsymbol{p}$-forms and duality equations. It has been shown in [4] that the tensor/vector system described by (3.12) can be extended on-shell to include higher order $p$-forms that are related by first-order duality equations to the physical fields. Let us briefly discuss how this extension is modified in the presence of hypermultiplets.

The $p$-form field content of (3.12) is given by vector and tensor fields $A_{\mu}{ }^{r}$ and $B_{\mu \nu}{ }^{I}$ and the three-form potentials $C_{\mu \nu \rho r}$ which however only appear under projection with the tensor $h^{r}{ }_{I}$. In a first step, the model may be extended to the full set of three-forms $C_{\mu \nu \rho r}$, as well as four-form potentials $C_{\mu \nu \rho \sigma \mathfrak{m}}^{(4)}$. Supersymmetry and gauge transformations of the former are given by

$$
\begin{aligned}
\Delta C_{\mu \nu \rho r} & =-2 d_{J r s}\left(\bar{\epsilon} \gamma_{\mu \nu \rho} \lambda^{s} \phi^{J}\right) \\
\Delta C_{\mu \nu \rho r} & =3 D_{[\mu} \Lambda_{\nu \rho] r}+6 d_{\text {Irs }} \mathcal{F}_{[\mu \nu}^{s} \Lambda_{\rho]}^{I}+2 d_{\text {Irs }} \mathcal{H}_{\mu \nu \rho}^{I} \Lambda^{s}-\theta_{r}{ }^{\mathfrak{m}} \Lambda_{\mu \nu \rho \mathfrak{m}},
\end{aligned}
$$

respectively. Here, $\theta_{r}{ }^{\mathfrak{m}}$ is the embedding tensor of (3.15) and $\Lambda_{\mu \nu \rho \mathfrak{m}}$ is the gauge parameter of the four-form potentials. Closing the supersymmetry algebra on the three-form potentials leads to the field equations (3.18). The hypermultiplet contribution of (3.18) is now absorbed into the resulting Stückelberg transformation $\Lambda_{\mu \nu \rho \mathfrak{m}}$ on the three-form potential which explains the appearance of the embedding tensor $\theta_{r}{ }^{\mathfrak{m}}$ in (3.21). Furthermore, closure of the supersymmetry algebra implies the first-order duality equation

$$
2 d_{\text {Irs }}\left(\mathcal{F}_{\mu \nu}^{s} \phi^{I}-2 \bar{\lambda}^{s} \gamma_{\mu \nu} \chi^{I}\right)=\frac{1}{4 !} \varepsilon_{\mu \nu \lambda \rho \sigma \tau} \mathcal{H}_{r}^{(4) \lambda \rho \sigma \tau} .
$$


where the field strength $\mathcal{H}_{r}^{(4)}$ is defined as

$$
\begin{aligned}
\mathcal{H}_{r}^{(4)}=D C_{r}+\theta_{r}{ }^{\mathfrak{m}} C_{\mathfrak{m}}^{(4)}-2 d_{\text {Irs }}( & \mathcal{F}^{s} \wedge B^{I}-\frac{1}{2} h^{s}{ }_{J} B^{I} \wedge B^{J} \\
& \left.+\frac{1}{3} d^{I}{ }_{p q} A^{s} \wedge K^{r q}+\frac{1}{36} f_{u v}{ }^{q} d^{I}{ }_{p q} A^{s} \wedge A^{p} \wedge A^{u} \wedge A^{v}\right),
\end{aligned}
$$

with $K^{r q}$ being the Chern-Simons form. In turn, it satisfies the Bianchi identity

$$
D \mathcal{H}_{r}^{(4)}=-2 d_{\text {Irs }} \mathcal{F}^{s} \wedge \mathcal{H}^{I}+\theta_{r}{ }^{\mathfrak{m}} \mathcal{H}_{\mathfrak{m}}^{(5)},
$$

with $\mathcal{H}_{\mathfrak{m}}^{(5)}$ denoting the field strength of the four-form potentials $C_{\mathfrak{m}}^{(4)}$. Equation (3.22) is the non-abelian first-order duality equation that relates the three-form potentials $C_{\mu \nu \rho r}$ to the vector fields and unlike the field equations (3.18)-(3.20) has no contributions from the hypermultiplet. Nevertheless, its derivative is precisely compatible with the second-order Yang-Mills equation (3.20), provided the field strength $\mathcal{H}_{\mathfrak{m}}^{(5)}$ in turn satisfies the first-order duality equation

$$
\begin{aligned}
\frac{1}{5 !} \varepsilon_{\mu \nu \rho \lambda \sigma \tau} \theta_{r}{ }^{\mathfrak{m}} \mathcal{H}_{\mathfrak{m}}^{(5) \nu \rho \lambda \sigma \tau}= & {\left[\left(X_{r}\right)_{I J}\left(\phi^{I} D_{\mu} \phi^{J}-2 \bar{\chi}^{I} \gamma_{\mu} \chi^{J}\right)+4\left(X_{r}\right)_{u}{ }^{s} d_{I s v} \phi^{I} \bar{\lambda}^{u} \gamma_{\mu} \lambda^{v}\right] } \\
& +\frac{2}{\lambda} \theta_{r}^{\mathfrak{m}}\left(X_{(\mathfrak{m}) \alpha} D_{\mu} q^{\alpha}-\bar{\psi}_{a} \gamma_{\mu} t_{(\mathfrak{m})}{ }_{b}{ }_{b} \psi^{b}\right)
\end{aligned}
$$

relating the four-form potentials $C_{\mathfrak{m}}^{(4)}$ to the scalar fields of the model (including hypers). Accordingly, the supersymmetry transformation rule for the 4 -form potentials in presence of hypermultiplets is modified to

$$
\theta_{r}{ }^{\mathfrak{m}} \Delta C_{\mu \nu \rho \sigma \mathfrak{m}}=\left(X_{r}\right)_{I J} \phi^{[I} \bar{\epsilon} \gamma_{\mu \nu \rho \sigma} \chi^{J]}-\frac{2}{\lambda} \theta_{r}^{\mathfrak{m}} X_{(\mathfrak{m})}^{\alpha} f^{i a}{ }_{\alpha} \bar{\epsilon}_{i} \gamma_{\mu \nu \rho \sigma} \psi_{a}
$$

combining contributions from tensor and hypermultiplets.

Superconformal symmetries. We conclude with a presentation of the superconformal symmetry transformations [35]. Denoting the fields in the theory by $\Phi=$ $\left(\phi^{I}, B_{\mu \nu}^{I}, \chi^{I}, A_{\mu}^{r}, Y^{i j}, \lambda^{r}, C_{\mu \nu \rho r}\right)$, the conformal transformations are given by

$$
\delta_{C} \Phi=\mathcal{L}_{\xi} \Phi+\lambda_{D} \Omega \Phi
$$

where $\mathcal{L}_{\xi}$ is the Lie derivative with respect to the conformal Killing vector defined by $\partial_{(\mu} \xi_{\nu)}=\Omega \eta_{\mu \nu}$, which also defines $\Omega$, and $\lambda_{D}$ are the Weyl weight for $\Phi$ given by $(2,0,5 / 2,0,2,3 / 2,0)$. The Lie derivative for the fermionic fields $\Psi=\left(\psi^{a}, \chi^{I}, \lambda^{r}\right)$, in particular, takes the form $\mathcal{L}_{\xi} \Psi=\xi^{\mu} \partial_{\mu} \Psi+\frac{1}{4} \partial_{\mu} \xi_{\nu} \gamma^{\mu \nu} \Psi$. On the other hand, the hypermultiplet fields transform as

$$
\begin{aligned}
\delta_{C} q^{\alpha} & =\mathcal{L}_{\xi} q^{\alpha}+4 \Omega \chi^{\alpha}, \\
\delta_{C} \psi^{a} & =\mathcal{L}_{\xi} \psi^{a}+\frac{5}{2} \Omega \psi^{a}-\Omega \chi^{\alpha} \omega_{\alpha}{ }^{a}{ }_{b} \psi^{b},
\end{aligned}
$$

with the homothetic Killing vector and spin connection from (2.3) and (3.1), respectively. The conformal supersymmetry transformations, on the other hand, involve conformal 
Killing spinors, consisting of a pair $\left(\eta_{+}, \eta_{-}\right)$that satisfy $\partial_{\mu} \eta_{+}-\frac{1}{2} \gamma_{\mu} \eta_{-}=0$. The superconformal transformations take the form of supersymmetry transformations in which the constant supersymmetry parameter $\epsilon$ is replaced by $\eta_{+}$, and the parameter $\eta_{-}$arises only in $\delta_{\eta_{-}} \chi^{I}=-\frac{1}{2} \phi^{I} \eta_{-}$. Note that the bosonic conformal transformation together with supersymmetry ensures the full superconformal symmetry since the commutator of conformal boost with supersymmetry yields the special supersymmetry generator [43].

\section{Eliminating auxiliary fields}

In the previous sections we have coupled the hypermultiplet Lagrangian of [19] to the tensor/vector system of [4]. The non-trivial interactions between vector-, tensor- and hypermultiplets are reflected in equation (3.18)

$$
d_{I r s} \phi^{I} Y^{i j s}-\frac{1}{2 \lambda} \theta_{r}^{\mathfrak{m}} \mu_{(\mathfrak{m})}^{i j}=2 d_{I r s} \bar{\lambda}^{s(i} \chi^{j) I}
$$

for the auxiliary fields $Y^{i j s}$ of the Yang-Mills multiplet. In particular, eliminating the auxiliary fields will introduce non-trivial couplings between tensor- and hypermultiplets of the model.

In this section, we will further analyze the form and the consequences of equation (4.1) and derive the couplings induced by elimination of the auxiliary fields. The explicit form of (4.1) depends on the field content of the model and the particular choice of the constant tensors $d_{I r s}, \theta_{r}{ }^{\mathfrak{m}}$, parametrizing the model. As discussed above, these constant tensors are subject to a number of algebraic constraints derived from (3.11). A general class of solutions to these constraints has been constructed in [17] based on a semi-simple Lie algebra $\mathfrak{g}$ under which all fields transform in non-trivial representations. Explicitly, w.r.t. this algebra the vector and tensor multiplets split into

$$
\begin{aligned}
A_{\mu}^{r} \rightarrow\left(A_{\mu}^{\mathfrak{m}}, B_{\mu}^{\mathrm{A}}\right), & \lambda^{r} \rightarrow\left(\lambda^{\mathfrak{m}}, \nu^{\mathrm{A}}\right), & Y_{i j}^{r} \rightarrow\left(Y_{i j}^{\mathfrak{m}}, Z_{i j}^{\mathrm{A}}\right), \\
B_{\mu \nu}^{I} \rightarrow\left(B_{\mu \nu}^{\mathrm{A}}, C_{\mu \nu \mathrm{A}}\right), & \chi^{I} \rightarrow\left(\chi^{\mathrm{A}}, \zeta_{\mathrm{A}}\right), & \phi^{I} \rightarrow\left(\phi^{\mathrm{A}}, \varphi_{\mathrm{A}}\right),
\end{aligned}
$$

where indices $\mathfrak{m}$ and $\mathrm{A}$ refer to the adjoint and an arbitrary fixed representation $\mathcal{R}$ of $\mathfrak{g}$, respectively. The model thus combines $n_{\mathrm{V}}=\operatorname{dim} \mathfrak{g}+\operatorname{dim} \mathcal{R}$ vector multiplets and $n_{\mathrm{T}}=$ $2 \operatorname{dim} \mathcal{R}$ tensor multiplets. The non-vanishing components of the gauge invariant constant tensors that define the model (referred to as Type III in [17]) are

$$
\begin{array}{rlrl}
\eta_{\mathrm{A}}^{\mathrm{B}} & =\eta_{\mathrm{A}}^{\mathrm{B}}=\delta_{\mathrm{A}}^{\mathrm{B}}, & h^{\mathrm{B}}=\delta_{\mathrm{A}}^{\mathrm{B}} & \\
f_{\mathfrak{m A}}^{\mathrm{B}}=-\frac{1}{2}\left(T_{\mathfrak{m}}\right)_{\mathrm{A}}^{\mathrm{B}}, & f_{\mathfrak{m} \mathfrak{n}}^{\mathfrak{p}}, \\
d^{\mathrm{B}}{ }_{\mathfrak{m} \mathrm{A}}=\frac{1}{2}\left(T_{\mathfrak{m}}\right)_{\mathrm{A}}^{\mathrm{B}}, & d_{\mathrm{ABC}}=d_{(\mathrm{ABC})}, & d_{\mathrm{AB \mathfrak {m }}}=d_{(\mathrm{AB}) \mathfrak{m}}, \quad d_{\mathrm{A} \mathfrak{m} \mathfrak{n}} .
\end{array}
$$

Here, $f_{\mathfrak{m} \mathfrak{n}}{ }^{\mathfrak{p}}$, and $\left(T_{\mathfrak{m}}\right)_{\mathrm{A}}^{\mathrm{B}}$ denote the structure constants and representation matrices of $\mathfrak{g}$, respectively, and $d_{(\mathrm{ABC})}, d_{(\mathrm{AB}) \mathfrak{m}}, d_{\mathrm{Amn}}$, are $\mathfrak{g}$-invariant tensors with the indicated symmetry 
properties, which obviously exist only for particular choice of $\mathfrak{g}$ and $\mathcal{R}$. In particular, the cubic scalar potential of (3.12) is exclusively triggered by the constant tensor $d_{\mathrm{ABC}}$ :

$$
\mathcal{L}_{\text {pot }} \propto d_{\mathrm{ABC}} \phi^{\mathrm{A}} \phi^{\mathrm{B}} \phi^{\mathrm{C}}
$$

For the hypermultiplet couplings, we choose the embedding tensor $\theta_{r}{ }^{\mathfrak{m}}$ as

$$
\theta_{\mathfrak{m}}^{\mathfrak{n}}=\delta_{\mathfrak{m}}^{\mathfrak{n}}, \quad \theta_{\mathrm{A}}^{\mathfrak{n}}=0
$$

such that only the vector fields $A_{\mu}^{\mathfrak{m}}$ participate in the gauging, and the algebra $\mathfrak{g}$ is identified with the algebra of gauged isometries (2.8) in the hyper-sector. In contrast, the vector fields $B_{\mu}^{\mathrm{A}}$ as well as the tensors $C_{\mu \nu \mathrm{A}}$ can be eliminated from the Lagrangian by field redefinition (see [17] for details).

As we have discussed in the introduction, a particularly interesting class of models is supposed to describe a field content that can be regrouped into multiplets of $(2,0)$ supersymmetry. In particular, this requires that tensor- and hypermultiplets arise in the same representation (i.e. $n_{\mathrm{H}}=n_{\mathrm{T}}$ ), such that they may recombine into $(2,0)$ tensor multiplets. The algebra $\mathfrak{g}$ is embedded into the orthogonal group $\mathrm{SO}\left(n_{\mathrm{T}}\right)$ via the generators $\left(X_{\mathfrak{m}}\right)_{I J}$ from (3.10). Correspondingly, in this case its action in the hypermultiplet sector is realized via the embedding (2.19) (with $B=0$ ) into the $\mathrm{SO}\left(n_{\mathrm{T}}\right)$ subalgebra of isometries on the flat target space of hypermultiplets. Indeed, with this realization it follows that part of the structures such as the supersymmetry variation (3.26) combining tensor- and hyper-scalars can be embedded into the manifest $(2,0)$ form

$$
\Delta C_{\mu \nu \rho \sigma \mathfrak{m}}=\left(X_{\mathfrak{m}}\right)_{I J} \phi^{\hat{\imath} \hat{\jmath}, I} \bar{\epsilon}_{\hat{\imath}} \gamma_{\mu \nu \rho \sigma} \chi_{\hat{\jmath}}^{J}
$$

with $\operatorname{Sp}(2)$ R-symmetry indices $\hat{\imath}, \hat{\jmath}=1, \ldots, 4$, and the five scalar fields $\phi^{[\hat{\imath} \hat{\jmath}]}$ combining the tensor- and hyper-scalars. Truncating the $(2,0)$ susy parameter as $\epsilon^{\hat{\imath}} \rightarrow\left(\epsilon^{i}, \tilde{\epsilon}^{i}\right) \rightarrow\left(\epsilon^{i}, 0\right)$, equation (4.6) indeed reduces to (3.26).

In the context of the M5-brane dynamics, the most interesting models describe tensor multiplets in the adjoint representation of the gauge group, i.e. correspond to the choice $\mathcal{R}=\operatorname{Adj}_{\mathfrak{g}}$. For the rest of this section, we will study a slightly more general class corresponding to choosing $\mathcal{R}=\operatorname{Adj}_{\mathfrak{g}} \oplus \mathbf{1}$. The role of the extra singlet will become clear in the following. Explicitly thus, tensor multiplets split into

$$
\left(B_{\mu \nu}^{\mathfrak{m}}, \chi^{\mathfrak{m}}, \phi^{\mathfrak{m}}\right),\left(B_{\mu \nu}^{0}, \chi^{0}, \phi^{0}\right),\left(C_{\mu \nu \mathfrak{m}}, \zeta_{\mathfrak{m}}, \varphi_{\mathfrak{m}}\right),\left(C_{\mu \nu 0}, \zeta_{0}, \varphi_{0}\right) .
$$

Furthermore, for the constants in (4.3), we choose the non-vanishing $\mathfrak{g}$-invariant tensors

$$
\begin{aligned}
& d_{\mathrm{Am \mathfrak {m }}}:\left\{\begin{aligned}
c_{1} \delta_{\mathfrak{m} \mathfrak{n}} & \text { for } \mathrm{A}=0, \\
0 & \text { otherwise },
\end{aligned}\right. \\
& d_{\mathrm{AB \mathfrak {m }}}:\left\{\begin{aligned}
c_{2} \delta_{\mathfrak{m} \mathfrak{n}} & \text { for }(\mathrm{A}, \mathrm{B})=(0, \mathfrak{n}) \text { or }(\mathrm{A}, \mathrm{B})=(\mathfrak{n}, 0), \\
0 & \text { otherwise },
\end{aligned}\right.
\end{aligned}
$$


and set to zero all components of $d_{(\mathrm{ABC})}$. In particular, absence of $d_{(\mathrm{ABC})}$ implies that there is no (unstable) cubic potential (4.4) for the tensor scalars. The part of the total Lagrangian containing the auxiliary fields then takes the form (suppressing R-symmetry indices)

$$
\begin{aligned}
\mathcal{L}_{Y}=\operatorname{Tr}\left[Y[Z, \varphi]-c_{1} \phi^{0} Y^{2}-2 c_{2} \phi^{0} Y Z+\frac{2}{\lambda} \mu Y\right. \\
\left.-2 Y\{\bar{\nu}, \zeta\}+2 Z\{\bar{\lambda}, \zeta\}+4 c_{1} Y \bar{\lambda} \chi^{0}+4 c_{2} Y \bar{\nu} \chi^{0}+4 c_{2} Z \bar{\lambda} \chi^{0}\right],
\end{aligned}
$$

where all the fields are matrix valued and in the adjoint representation of $\mathfrak{g}$. The resulting field equations (4.1) for the auxiliary fields can be written as

$$
\begin{aligned}
{[Y, \varphi]+2 c_{2} \phi^{0} Y } & =J, \\
{[Z, \varphi]-2 \phi^{0}\left(c_{1} Y+c_{2} Z\right)+\frac{2}{\lambda} \mu } & =K,
\end{aligned}
$$

where $J$ and $K$ are bilinear in fermions which can be easily read off from (4.9). The form of these equations shows that for generic values of the parameters $c_{1,2}$, the auxiliary fields $Y$ and $Z$ are uniquely determined and can be eliminated from the Lagrangian. On the other hand, for $c_{1,2}=0$ only part of $Y$ and $Z$ is determined which implies constraints on the sources $J$ and $K$. To make this explicit, it is convenient to consider the Lie algebra commutators in the Cartan-Weyl basis, in which the generators are denoted by $\left(\vec{H}, E^{\alpha}, E^{-\alpha}\right)$. Furthermore let us take the field $\varphi$ to lie in the Cartan subalgebra as

$$
\varphi=\vec{\varphi} \cdot \vec{H}
$$

The non-vanishing commutators are

$$
\left[\vec{H}, E^{ \pm \alpha}\right]= \pm \vec{\alpha} E^{ \pm \alpha}, \quad\left[E^{\alpha}, E^{\beta}\right]=N_{\alpha \beta} E^{\alpha+\beta}, \quad\left[E^{\alpha}, E^{-\alpha}\right]=\frac{2}{|\alpha|^{2}} \vec{\alpha} \cdot \vec{H}
$$

where $\vec{\alpha}$ is the root vector and $N_{\alpha \beta}$ are numbers associated with the specific Lie algebra. Thus, expanding

$$
Y=\vec{Y} \cdot \vec{H}+Y_{\alpha} E^{\alpha}+Y_{-\alpha} E^{-\alpha}, \quad \text { idem } Z, J, K,
$$

from (4.10) it readily follows that ${ }^{5}$

$$
Y=\frac{1}{2 c_{2} \phi^{0}} \vec{J} \cdot \vec{H}+\frac{J_{\alpha} E^{\alpha}}{2 c_{2} \phi^{0}+\vec{\alpha} \cdot \vec{\varphi}}+\frac{J_{-\alpha} E^{-\alpha}}{2 c_{2} \phi^{0}-\vec{\alpha} \cdot \vec{\varphi}} .
$$

Solving (4.11) similarly and substituting the solution for $Y$ we then find

$$
\begin{aligned}
Z= & -\frac{1}{2 c_{2} \phi^{0}}\left(\vec{K}+2 c_{1} \phi^{0} \vec{J}-\frac{2}{\lambda} \vec{\mu}\right) \cdot \vec{H}-\frac{1}{2 c_{2} \phi^{0}-\vec{\alpha} \cdot \vec{\varphi}}\left(K_{\alpha}+\frac{2 c_{1} \phi^{0} J_{\alpha}}{2 c_{2} \phi^{0}+\vec{\alpha} \cdot \vec{\varphi}}-\frac{2}{\lambda} \mu_{\alpha}\right) E^{\alpha} \\
& -\frac{1}{2 c_{2} \phi^{0}+\vec{\alpha} \cdot \vec{\varphi}}\left(K_{-\alpha}+\frac{2 c_{1} \phi^{0} J_{-\alpha}}{2 c_{2} \phi^{0}-\vec{\alpha} \cdot \vec{\varphi}}-\frac{2}{\lambda} \mu_{-\alpha}\right) E^{-\alpha}
\end{aligned}
$$

\footnotetext{
${ }^{5}$ The fields $(Y, Z, J, K, \mu)$ are understood to be rotated by the similarity transformation that puts the scalar field $\varphi$ in the Cartan subalgebra in the Cartan-Weyl basis.
} 
For generic values of the constants $c_{1}, c_{2}$ the auxiliary fields are thus fully determined and can be eliminated from the Lagrangian. Let us note that due to the form of the couplings (4.8), elimination of the auxiliary fields $Y, Z$ does not introduce any bosonic potential for the hyperscalars (unlike for the standard YM-hyper couplings [19] where elimination of the auxiliary fields introduces a potential quadratic in the hyper-Kähler moment maps $\mu_{i j}$ ). The resulting moduli space for the scalars thus is not constrained by any potential.

On the other hand, if $c_{2}=0$, the auxiliary fields in the Cartan subalgebra remain undetermined and we find the constraints $\vec{J}=0$ and $\vec{K}=2 \vec{\mu} / \lambda$. In this case, using the expansions (4.14) with $\vec{Y}$ and $\vec{Z}$ as undetermined entries, and the remaining components from (4.15) and (4.16), and finally setting $c_{1}=0$ for simplicity, the Lagrangian (4.9) becomes

$$
\begin{aligned}
\mathcal{L}_{Y}= & -\vec{Y} \cdot\left(\vec{K}-\frac{2}{\lambda} \vec{\mu}\right)+\vec{Z} \cdot \vec{J} \\
& -\frac{2}{|\alpha|^{2} \vec{\alpha} \cdot \vec{\varphi}}\left[J_{\alpha}\left(K_{-\alpha}-\frac{2}{\lambda} \mu_{-\alpha}\right)-J_{-\alpha}\left(K_{\alpha}-\frac{2}{\lambda} \mu_{\alpha}\right)\right] .
\end{aligned}
$$

This exhibits the role of the undetermined auxiliary fields as Lagrange multipliers. In particular the constraint $\vec{\mu}=\frac{\lambda}{2} \vec{K}$ modifies the hyper-Kähler geometry and eliminates degrees of freedom from the hyper-sector. What we have shown in the above is that such constraints can precisely be avoided by introducing abelian factors among the tensor multiplets with the specific couplings (4.8). Let us finally note, that in the Lagrangian (3.12), the choice of (4.8) in particular gives rise to interaction terms of the form

$$
\mathcal{L}_{\phi \mathcal{F}^{2}}=-\frac{1}{2} c_{1} \eta_{\mathfrak{m} \mathfrak{n}} \phi^{0} F_{\mu \nu}^{\mathfrak{m}} F^{\mu \nu \mathfrak{n}}
$$

and thus to exactly those interactions that were taken into account for the anomaly cancelation conditions in [23]. This led to the selection of $A D E$ gauge groups. Here, we have seen that such couplings are naturally present in the theory.

\section{Conclusions}

In this paper, we have constructed six-dimensional superconformal models with non-abelian tensor and hypermultiplets. They comprise the field content of $(2,0)$ theories, coupled to $(1,0)$ vector multiplets. The hypermultiplets are described by gauged nonlinear sigma models with a hyper-Kähler cone target space and minimal coupling to the superconformal tensor/vector models of [4]. Elimination of the auxiliary fields from the vector multiplets then further induces non-trivial couplings between hyper and tensor multiplets. We have shown that proper elimination of the auxiliary fields requires abelian factors among the tensor multiplets but unlike standard YM-hyper couplings does not give rise to a scalar potential. Furthermore, elimination of the auxiliary fields provides couplings (4.18) that were previously considered for anomaly cancellations with abelian tensor multiplets and resulted in the selection of $A D E$ gauge groups. We have shown that on the level of the 
equations of motion, the system may be extended to include non-propagating three- and four-forms, related by a set of non-abelian first-order duality equations to the physical fields.

It remains an intriguing open question, how much of the presented structures can be carried over to $(2,0)$ supersymmetric theories. Whereas the tensor and hyper multiplets combine into the field content of $(2,0)$ tensor multiplets and exhibit some unifying structures such as (4.6), it is clear that the dynamical degrees of freedom from the propagating vector multiplets will have to be eliminated upon such a supersymmetry enhancement.

The other main open question is of course the quantization of the models, and the fate of the conformal symmetry at the quantum level. For superconformal hypermultiplet actions with and without higher derivative terms such questions have been addressed in [44, 45]. For the models presented here at the classical level, a key issue will be whether the ghost states resulting from the tensor sector of (3.12) can be decoupled with the help of the large extended tensor gauge symmetry. This may require to set up a proper Hamiltonian formalism for the self-dual tensor fields along the lines of $[46,47]$. Last but not least, the study of anomalies in the generalized gauge symmetries of the models we have presented here will be of great interest.

\section{Acknowledgments}

This work is supported in part by the Fédération de Physique André Marie Ampère. R.W. thanks K. Gawedzki and M. Rocek for useful discussions and the Simons Center for Geometry and Physics and the organizers of the Simons Summer Workshop in Mathematics and Physics 2012 for the stimulating environment, as well as the ENS Lyon for hospitality. E.S. would like to thank ENS Lyon for hospitality and Y. Pang and L. Wulff for helpful discussions. The research of E.S. is supported in part by NSF grant PHY-0906222.

\section{A Conventions}

Indices. In the main text different kind of indices appear which are collect in table 1. The vector representation of $\operatorname{Sp}(1)$ is usually denoted by an arrow (occasionally indices $\mathrm{i}, \mathrm{j}=1,2,3$ are used), or given in the bi-spinor notation,

$$
x_{j}^{i}:=i \vec{\sigma}_{j}{ }_{j} \vec{x}, \quad x^{i j}=\varepsilon^{j k} x_{k}^{i} \quad \Rightarrow \quad\left(x^{i j}\right)^{*}=\varepsilon_{i k} \varepsilon_{j \ell} x^{k \ell}=x_{i j},
$$

with $\varepsilon^{i k} \varepsilon_{j k}=\delta_{j}^{i}$ and $\varepsilon^{12}=\varepsilon_{12}=1$.

Spinors. We work with a flat world-volume metric of signature $(-+++++)$ and LeviCivita tensor $\varepsilon_{012345}=1,\left\{\gamma_{\mu}, \gamma_{\nu}\right\}=2 \eta_{\mu \nu}$ and $\gamma_{7}:=\gamma_{012345}$. The spinor chiralities are given by

$$
\gamma_{7} \epsilon^{i}=\epsilon^{i}, \quad \gamma_{7} \lambda^{i r}=\lambda^{i r}, \quad \gamma_{7} \chi^{i I}=-\chi^{i I}, \quad \gamma_{7} \psi^{a}=-\psi^{a} .
$$

For $\operatorname{Sp}(1)$ indices we use standard standard northwest-southeast conventions according to $\epsilon^{i}=\varepsilon^{i j} \epsilon_{j}, \epsilon_{i}=\epsilon^{j} \varepsilon_{j i}$, etc. , and analogously for the $\operatorname{Sp}(n)$ indices, i.e. $\psi^{a}=\Omega^{a b} \psi_{b}, \psi_{a}=$ $\psi^{b} \Omega_{b a}$. All spinors satisfy a symplectic Majorana condition,

$$
\bar{\epsilon}_{i}:=\epsilon_{i}^{T} C=\left(\epsilon^{i}\right)^{\dagger} i \gamma^{0}, \quad \bar{\psi}_{a}:=\psi_{a}^{T} C=\left(\psi_{a}\right)^{\dagger} i \gamma^{0},
$$




\begin{tabular}{|l|c|l|}
\hline Label & Range & Comment \\
\hline$\mu, \nu, \ldots$ & $0, \ldots, 5$ & world-volume Lorentz indices \\
$\alpha, \beta, \ldots$ & $1, \ldots 4 n$ & real target space coordinates \\
$a, b, \ldots$ & $1, \ldots, 2 n$ & $\mathrm{Sp}(n)$ indices, complex coordinates \\
$i, j, \ldots$ & 1,2 & $\mathrm{Sp}(1)$ indices \\
$\hat{\mathfrak{m}}, \hat{\mathfrak{n}}, \ldots$ & $1, \ldots, \operatorname{dim}(\hat{G})$ & isometries of $\mathcal{Q}_{4(n-1)}$ \\
$\mathfrak{m}, \mathfrak{n}, \ldots$ & $1, \ldots, \operatorname{dim}(G)$ & gauged isometries of $\mathcal{Q}_{4(n-1)}$ \\
$I, J, \ldots$ & $1, \ldots, n_{\mathrm{T}}$ & tensor multiplets \\
$r, s, \ldots$ & $1, \ldots, n_{\mathrm{V}}$ & vector multiplets \\
\hline
\end{tabular}

Table 1. Conventions for the various indices.

where the charge conjugation matrix satisfy $\gamma_{\mu}^{T}=-C \gamma_{\mu} C^{-1}$. Note that indices are exclusively raised/lowered with the symplectic forms, i.e. $\bar{\epsilon}^{i}=-\left(\epsilon_{i}\right)^{\dagger} i \gamma^{0}$ etc.. The same relations hold for $\lambda^{i r}$ and $\chi^{i I}$. We refer to the appendix of [4] for further useful relations.

\section{B Target space geometry}

We collect here some basic relations for the geometrical quantities of the HK target space. Many of the following relations are the six-dimensional versions of the ones given in [20].

Vielbeine. The $\operatorname{Sp}(n)$ vielbeine and connection are defined in (3.1), such that metric takes the flat form (2.17). Thus for the special case of a flat target space, i.e. $g_{\alpha \beta}=\delta_{\alpha \beta}$, the explicit form of the vielbeine is given by $\left(\kappa^{2}=i\right)$,

$$
\left[\begin{array}{l}
f^{1 a}{ }_{\alpha} \\
f^{2 a}{ }_{\alpha}
\end{array}\right]=\frac{\kappa}{\sqrt{2}}\left[\begin{array}{cc}
\Omega & -i \Omega \\
-i \mathbb{1} & \mathbb{1}
\end{array}\right], \quad\left[f^{\alpha}{ }_{1 a} f^{\alpha}{ }_{2 a}\right]=\frac{\bar{\kappa}}{\sqrt{2}}\left[\begin{array}{cc}
-\Omega & i \mathbb{1} \\
-i \Omega & \mathbb{1}
\end{array}\right] .
$$

Besides the metric also the hypercomplex structure and Kähler forms (2.1) can be expressed in terms of the vielbeine:

$$
\omega_{\alpha \beta}^{i j}=2 \Omega_{a b} f^{(i a}{ }_{\alpha} f^{j) b} b_{\beta} \quad, \quad \vec{J}_{\beta}^{\alpha}=-i f^{\alpha}{ }_{i a} \vec{\sigma}^{i}{ }_{j} f^{j a}{ }_{\beta},
$$

where for the constant flat space vielbeine the first relation reduces to the expression given in (2.17). With this the orthogonality of the vielbeine can be written as,

$$
f^{i a}{ }_{\alpha} f^{\alpha}{ }_{j b}=\delta^{i}{ }_{j} \delta_{b}^{a} \quad, \quad f^{\alpha}{ }_{j a} f_{\beta}^{i a}=\frac{1}{2}\left(\delta^{i}{ }_{j} \delta_{\beta}^{\alpha}+i \vec{\sigma}^{i}{ }_{j} \vec{J}_{\beta}{ }_{\beta}\right),
$$

and they satisfy the pseudo reality condition $\left(f^{i a}{ }_{\alpha}\right)^{*}=\varepsilon_{i j} \Omega_{a b} f^{j b}{ }_{\alpha}$. With the definition of the connection (2.17) this gives,

$$
\omega_{\alpha}^{a} b=\Omega^{a c} \omega_{\alpha}^{d}{ }_{c} \Omega_{d b}=-\left(\omega_{\alpha}^{b} a\right)^{*} \quad \Rightarrow \quad\left(\Omega \omega_{\alpha}\right)_{[a b]}=0 .
$$

Hence the connection coefficients are $\operatorname{Sp}(n)$ matrices (2.14). 
Curvatures. The curvatures of the Levi-Civita and $\operatorname{Sp}(n)$ connection are given by

$$
R_{\delta \alpha \beta}^{\gamma}=2\left(\partial_{[\alpha} \Gamma_{\beta] \delta}^{\gamma}+\Gamma_{[\alpha \mid \epsilon}^{\gamma} \Gamma_{\beta] \delta}^{\epsilon}\right), \quad \mathcal{R}_{b \alpha \beta}^{a}=2\left(\partial_{[\alpha} \omega_{\beta]}{ }^{a} b+\omega_{[\alpha}{ }^{a}{ }_{c} \omega_{\beta]}{ }^{c} b\right)
$$

The integrability condition of the vielbein postulate (3.1) implies that these curvature are related in the following way,

$$
R_{\delta \alpha \beta}^{\delta}=f^{\gamma}{ }_{i a} f_{\delta}^{i b} \mathcal{R}_{b \alpha \beta}^{a}, \quad \delta_{i}^{i} \mathcal{R}_{b \alpha \beta}^{a}=f^{i a}{ }_{\gamma} f_{j b}^{\delta} R^{\gamma}{ }_{\delta \alpha \beta} .
$$

The symmetries of the Riemann tensor and the first Bianchi identity further imply,

$$
f^{\alpha}{ }_{i a} f^{\beta}{ }_{j b} f^{\gamma}{ }_{k c} f^{\delta}{ }_{\ell d} R_{\alpha \beta \gamma \delta}=\varepsilon_{i j} \varepsilon_{k l} W_{a b c d}, \quad f^{\gamma}{ }_{k c} f^{\delta}{ }_{\ell d} \mathcal{R}_{a b \gamma \delta}=-\varepsilon_{k \ell} W_{a b c d},
$$

where $W_{a b c d}=W_{(a b c d)}$ is the totally symmetric curvature tensor, with reality property $\left(W_{a b c d}\right)^{*}=W^{a b c d}$, that appears in the Lagrangian (3.3). $\operatorname{Sp}(n)$ indices are raised/lowered as described in appendix A. From the second Bianchi identity it follows that

$$
\nabla_{[\alpha} R_{\beta \gamma] \delta \epsilon}=0 \Rightarrow f^{\alpha}{ }_{i a} \mathcal{D}_{\alpha} W_{b c d e}=f^{\alpha}{ }_{i(a} \mathcal{D}_{\alpha} W_{b c d e)},
$$

where $\mathcal{D}_{\alpha}$ is the covariant derivative w.r.t. the $\operatorname{Sp}(n)$ connection.

Isometries. The vielbeine introduced here are adjusted to the $\mathrm{H}_{\mathrm{Sp}(1)} \otimes \mathrm{P}_{\mathrm{Sp}(n)}$ structure of the tangent space (3.1). They therefore relate the coordinate basis to vector fields of the form,

$$
e_{i a}=\theta_{i} \otimes e_{a}=f^{\alpha}{ }_{i a} \partial_{\alpha},
$$

with an analogous relation for the dual basis with the inverse vielbein. The Lie derivative of these vector fields along a vector field $X$ is then given by,

$$
\mathscr{L}_{X} e_{i a}=\left[\delta^{j}{ }_{i}\left(X^{\alpha} \omega_{\alpha}{ }^{b} b-t^{b}{ }_{a}\right)-\frac{1}{2} \vec{\sigma}^{j}{ }_{i} \vec{t}^{b}{ }_{a}\right] e_{j b}, \quad t^{b}{ }_{a}=\frac{1}{2} f^{i b}{ }_{\alpha}\left(\nabla_{\beta} X^{\alpha}\right) f^{\beta}{ }_{i a},
$$

where $t^{b}{ }_{a}$ was introduced below (3.4) and $\vec{t}^{b}{ }_{a}=f^{\beta}{ }_{a} \vec{\sigma} f^{b}{ }_{\alpha}\left(\nabla_{\beta} X^{\alpha}\right)$. For diffeomorphism or isometries that commute $\mathrm{Sp}(1)$ isometries (2.4), and thus preserve the $\mathrm{H}_{\mathrm{Sp}(1)} \otimes \mathrm{P}_{\mathrm{Sp}(n)}$ structure, the latter matrices vanish. In that case one has,

$$
\mathscr{L}_{X} e_{a}=\left(X^{\alpha} \omega_{\alpha}^{b}{ }^{b}-t^{b}{ }_{a}\right) e_{b} \quad \Rightarrow \quad \mathscr{L}_{X} W_{a}=X^{\alpha} \mathcal{D}_{\alpha} W_{a}+t^{b}{ }_{a} W_{b} \quad \text { etc. }
$$

In the case that $X$ is also an isometry, $\nabla_{(\alpha} X_{\beta)}=0$ one finds,

$$
\Omega^{a c} t^{d}{ }_{c} \Omega_{d b}=t^{a}{ }_{b}=-\left(t^{b}{ }_{a}\right)^{*} \quad \Rightarrow \quad(\Omega t)_{[a b]}=0,
$$

These relations are the same as for the $\operatorname{Sp}(n)$ connection (B.4). In the case that the isometries $X_{(\mathfrak{m})}$ obey $\left[X_{(\mathfrak{m})}, X_{(\mathfrak{n})}\right]=-f_{\mathfrak{m n}}{ }^{\mathfrak{p}} X_{(\mathfrak{p})}$ one also finds,

$$
\mathcal{D}_{\alpha} t_{(\mathfrak{m})}{ }_{b}{ }_{b}=\mathcal{R}_{b \alpha \beta}^{a} X_{(\mathfrak{m})}^{\beta}, \quad\left[t_{(\mathfrak{m})}, t_{(\mathfrak{n})}\right]_{b}^{a}=f_{\mathfrak{m n}}{ }^{\mathfrak{p}} t_{(\mathfrak{p})}{ }^{a}{ }_{b}+\mathcal{R}^{a}{ }_{b \alpha \beta} X_{(\mathfrak{m})}^{\alpha} X_{(\mathfrak{n})}^{\beta} .
$$

We finally mention the equivariance condition for the moment maps of triholomorphic isometries $X_{(\mathfrak{m})}(2.7)$. The identity $i_{\left[X_{(\mathfrak{m})}, X_{(\mathfrak{n})}\right]} \vec{\omega}=\left[\mathscr{L}_{X_{(\mathfrak{m})}}, i_{X_{(\mathfrak{n})}}\right] \vec{\omega}$ implies for triholomorphic isometries,

$$
\mathscr{L}_{X(\mathfrak{m})} \vec{\mu}_{\mathfrak{n}}=\vec{\omega}_{\alpha \beta} X_{(\mathfrak{m})}^{\alpha} X_{(\mathfrak{n})}^{\beta}=-f_{\mathfrak{m n}} \vec{\mu}_{\mathfrak{p}}+\text { const. }
$$

where superconformal symmetry fixes the constant to be zero. 
Open Access. This article is distributed under the terms of the Creative Commons Attribution License which permits any use, distribution and reproduction in any medium, provided the original author(s) and source are credited.

\section{References}

[1] W. Nahm, Supersymmetries and their representations, Nucl. Phys. B 135 (1978) 149 [INSPIRE].

[2] E. Witten, String theory dynamics in various dimensions, Nucl. Phys. B 443 (1995) 85 [hep-th/9503124] [INSPIRE].

[3] X. Bekaert, M. Henneaux and A. Sevrin, Chiral forms and their deformations, Commun. Math. Phys. 224 (2001) 683 [hep-th/0004049] [INSPIRE].

[4] H. Samtleben, E. Sezgin and R. Wimmer, $(1,0)$ superconformal models in six dimensions, JHEP 12 (2011) 062 [arXiv: 1108.4060] [INSPIRE].

[5] B. de Wit and H. Samtleben, Gauged maximal supergravities and hierarchies of nonAbelian vector-tensor systems, Fortsch. Phys. 53 (2005) 442 [hep-th/0501243] [INSPIRE].

[6] B. de Wit, H. Nicolai and H. Samtleben, Gauged supergravities, tensor hierarchies and M-theory, JHEP 02 (2008) 044 [arXiv:0801.1294] [INSPIRE].

[7] A. Kotov and T. Strobl, Characteristic classes associated to Q-bundles, arXiv:0711.4106 [INSPIRE].

[8] J.C. Baez and J. Huerta, An invitation to higher gauge theory, Gen. Rel. Grav. 43 (2011) 2335.

[9] A. Kotov and T. Strobl, Generalizing geometry - Algebroids and sigma models, in Handbook of pseudo-Riemannian geometry and supersymmetry, V. Cortes ed., IRMA Lect. Math. Theor. Phys. volume 16, European Mathematical Sociecty, Zürich Switzerland (2010).

[10] D. Fiorenza, H. Sati and U. Schreiber, Multiple M5-branes, string 2-connections and $7 D$ nonabelian Chern-Simons theory, arXiv:1201.5277 [INSPIRE].

[11] C. Sämann and M. Wolf, Non-abelian tensor multiplet equations from twistor space, arXiv:1205.3108 [INSPIRE].

[12] S. Palmer and C. Sämann, M-brane models from non-abelian gerbes, JHEP 07 (2012) 010 [arXiv: 1203.5757] [INSPIRE].

[13] C.-S. Chu, A theory of non-abelian tensor gauge field with non-abelian gauge symmetry $G \times G$, arXiv:1108.5131 [INSPIRE].

[14] J. Bagger and N. Lambert, Gauge symmetry and supersymmetry of multiple M2-branes, Phys. Rev. D 77 (2008) 065008 [arXiv:0711.0955] [InSPIRE].

[15] A. Gustavsson, Algebraic structures on parallel M2-branes, Nucl. Phys. B 811 (2009) 66 [arXiv:0709.1260] [INSPIRE].

[16] O. Aharony, O. Bergman, D.L. Jafferis and J. Maldacena, $N=6$ superconformal Chern-Simons-matter theories, M2-branes and their gravity duals, JHEP 10 (2008) 091 [arXiv:0806.1218] [INSPIRE]. 
[17] H. Samtleben, E. Sezgin, R. Wimmer and L. Wulff, New superconformal models in six dimensions: Gauge group and representation structure, PoS(CORFU2011) 071 [arXiv: 1204.0542] [INSPIRE].

[18] M. Akyol and G. Papadopoulos, $(1,0)$ superconformal theories in six dimensions and Killing spinor equations, JHEP 07 (2012) 070 [arXiv: 1204.2167] [INSPIRE].

[19] G. Sierra and P. Townsend, The gauge invariant $N=2$ supersymmetric $\sigma$-model with general scalar potential, Nucl. Phys. B 233 (1984) 289 [INSPIRE].

[20] B. de Wit, B. Kleijn and S. Vandoren, Superconformal hypermultiplets, Nucl. Phys. B 568 (2000) 475 [hep-th/9909228] [INSPIRE].

[21] B. de Wit, M. Roček and S. Vandoren, Gauging isometries on hyperKähler cones and quaternion Kähler manifolds, Phys. Lett. B 511 (2001) 302 [hep-th/0104215] [INSPIRE].

[22] J.A. Wolf, Complex homogeneous contact manifolds and quaternionic symmetric spaces, $J$. Math. Mech. 14 (1965) 1033.

[23] J.D. Blum and K.A. Intriligator, New phases of string theory and $6 D R G$ fixed points via branes at orbifold singularities, Nucl. Phys. B 506 (1997) 199 [hep-th/9705044] [INSPIRE].

[24] N. Seiberg and E. Witten, Comments on string dynamics in six-dimensions, Nucl. Phys. B 471 (1996) 121 [hep-th/9603003] [INSPIRE].

[25] M. Duff, H. Lü and C. Pope, Heterotic phase transitions and singularities of the gauge dyonic string, Phys. Lett. B 378 (1996) 101 [hep-th/9603037] [INSPIRE].

[26] N. Lambert and P. Richmond, $(2,0)$ supersymmetry and the light-cone description of M5-branes, JHEP 02 (2012) 013 [arXiv:1109.6454] [INSPIRE].

[27] F. Bonetti, T.W. Grimm and S. Hohenegger, Non-abelian tensor towers and $(2,0)$ superconformal theories, arXiv:1209.3017 [INSPIRE].

[28] B. Czech, Y.-t. Huang and M. Rozali, Amplitudes for multiple M5 branes, JHEP 10 (2012) 143 [arXiv:1110.2791] [INSPIRE].

[29] J. Rosseel and A. Van Proeyen, Hypermultiplets and hypercomplex geometry from 6 to 3 dimensions, Class. Quant. Grav. 21 (2004) 5503 [hep-th/0405158] [INSPIRE].

[30] M. Chiodaroli, M. Gunaydin and R. Roiban, Superconformal symmetry and maximal supergravity in various dimensions, JHEP 03 (2012) 093 [arXiv:1108.3085] [INSPIRE].

[31] W.D. Linch, III and G. Tartaglino-Mazzucchelli, Six-dimensional supergravity and projective superfields, JHEP 08 (2012) 075 [arXiv: 1204.4195] [INSPIRE].

[32] E. Bergshoeff et al., The map between conformal hypercomplex/hyper-Kähler and quaternionic(-Kähler) geometry, Commun. Math. Phys. 262 (2006) 411 [hep-th/0411209] [INSPIRE].

[33] L. Álvarez-Gaumé and D.Z. Freedman, Geometrical structure and ultraviolet finiteness in the supersymmetric $\sigma$-model, Commun. Math. Phys. 80 (1981) 443 [INSPIRE].

[34] G. Sierra and P. Townsend, The hyper-Kähler supersymmetric $\sigma$-model in six-dimensions, Phys. Lett. B 124 (1983) 497 [INSPIRE].

[35] E. Sezgin and Y. Tanii, Superconformal $\sigma$-models in higher than two-dimensions, Nucl. Phys. B 443 (1995) 70 [hep-th/9412163] [INSPIRE].

[36] A. Swann, Hyper-Kähler and quaternionic Kähler geometry, Math. Ann. 289 (1991) 421. 
[37] J. Bagger and E. Witten, Matter couplings in $N=2$ supergravity, Nucl. Phys. B 222 (1983) 1 [INSPIRE].

[38] B. de Wit, M. Roček and S. Vandoren, Hypermultiplets, hyper-Kähler cones and quaternion Kähler geometry, JHEP 02 (2001) 039 [hep-th/0101161] [INSPIRE].

[39] C. LeBrun and S. Salamon, Strong rigidity of positive quaternion-Kähler manifolds, Invent. Math. 118 (1994) 109.

[40] K. Shankar, Isometry groups of homogeneous spaces with positive sectional curvature, DiffGeom. Appl. 14 (2001) 57.

[41] C. Hull, A. Karlhede, U. Lindström and M. Roček, Nonlinear $\sigma$-models and their gauging in and out of superspace, Nucl. Phys. B 266 (1986) 1 [INSPIRE].

[42] J.A. Bagger, Supersymmetric sigma models, in Supersymmetry, K. Dietz et al. eds., NATO Adv. Sci. Inst. Ser. B Phys. colume 125, Plenum Press, New York U.S.A. (1985).

[43] E. Bergshoeff, E. Sezgin and A. Van Proeyen, Superconformal tensor calculus and matter couplings in six-dimensions, Nucl. Phys. B 264 (1986) 653 [Erratum ibid. B 598 (2001) 667] [INSPIRE].

[44] E. Ivanov, A.V. Smilga and B. Zupnik, Renormalizable supersymmetric gauge theory in six dimensions, Nucl. Phys. B 726 (2005) 131 [hep-th/0505082] [INSPIRE].

[45] E. Ivanov and A.V. Smilga, Conformal properties of hypermultiplet actions in six dimensions, Phys. Lett. B 637 (2006) 374 [hep-th/0510273] [INSPIRE].

[46] M. Henneaux and C. Teitelboim, Dynamics of chiral (selfdual) $p$ forms, Phys. Lett. B 206 (1988) 650 [INSPIRE].

[47] P. Pasti, D.P. Sorokin and M. Tonin, On Lorentz invariant actions for chiral $p$ forms, Phys. Rev. D 55 (1997) 6292 [hep-th/9611100] [INSPIRE]. 